\title{
IdeAs
}

Idées d'Amériques

$17 \mid 2021$

Villes et culture dans les Amériques

\section{Un Voyage à travers Cinq Décennies de Lutte pour l'Autodétermination des Peuples Autochtones des Amériques : Entretien avec Armstrong A. Wiggins}

\section{Laetitia Braconnier-Moreno et Laura Cahier}

Traducteur : Laetitia Braconnier-Moreno et Laura Cahier

\section{OpenEdition}

\section{Journals}

Édition électronique

URL : https://journals.openedition.org/ideas/11163

DOI : $10.4000 /$ ideas. 11163

ISSN : 1950-5701

\section{Éditeur}

Institut des Amériques

\section{Référence électronique}

Laetitia Braconnier-Moreno et Laura Cahier, « Un Voyage à travers Cinq Décennies de Lutte pour I'Autodétermination des Peuples Autochtones des Amériques : Entretien avec Armstrong A. Wiggins ", IdeAs [En ligne], 17 | 2021, mis en ligne le 01 mars 2020, consulté le 06 juin 2021. URL : http:// journals.openedition.org/ideas/11163; DOI : https://doi.org/10.4000/ideas.11163

Ce document a été généré automatiquement le 6 juin 2021.

\section{cc) (1)}

IdeAs - Idées d'Amériques est mis à disposition selon les termes de la licence Creative Commons Attribution - Pas d'Utilisation Commerciale - Pas de Modification 4.0 International. 


\title{
Un Voyage à travers Cinq Décennies de Lutte pour l'Autodétermination des Peuples Autochtones des Amériques : Entretien avec Armstrong A. Wiggins
}

\author{
Laetitia Braconnier-Moreno et Laura Cahier
}

Traduction : Laetitia Braconnier-Moreno et Laura Cahier

\section{Introduction}

1 En résonnances avec le Dossier éclairage sur les «leaders sociaux en Colombie » publié dans le $n^{\circ} 17$ de la revue IdeAs, Laetitia Braconnier Moreno et Laura Cahier ont rencontré Armstrong A. Wiggins afin de discuter de son parcours comme leader autochtone et défenseur des droits humains.

2 Né au Nicaragua, il est un Indien ${ }^{1}$ Mískito du village de Karatá, La Moskitia. À l'âge de dix-huit ans, il devient président de sa communauté Karatá; en 1972, il fonde l'AIPROMISU, la première organisation indienne régionale de La Moskitia dont l'objectif est de défendre les droits des Mískitos à l'autodétermination, à la terre, aux territoires, à l'environnement, aux ressources naturelles, au développement durable, et à la survie culturelle. En 1977, Armstrong et d'autres dirigeants autochtones du monde entier se rendent au siège des Nations Unies à Genève afin de demander à la communauté internationale de tenir les États responsables pour les violations de leurs droits individuels et collectifs. Après la révolution sandiniste de 1979 au Nicaragua, Armstrong devient le représentant national de MISURASATA, une organisation régionale indienne. En raison de son travail en faveur des droits humains à La Moskitia, il est fait prisonnier politique sous les régimes de Somoza et des sandinistes. En 1981, la situation politique au Nicaragua le contraint à l'exil. Une fois aux États-Unis, 
Armstrong commence à travailler pour l'Indian Law Resource Center (ILRC) où il occupe actuellement le poste de Directeur du bureau de Washington D.C. Au cours des deux dernières décennies, Armstrong a participé activement à de multiples affaires portant sur les droits humains de nombreux Peuples autochtones des Amériques, notamment auprès de la Commission interaméricaine des droits de l'homme et à la Cour interaméricaine des droits de l'homme.

En raison de la pandémie de la Covid-19, cet entretien a eu lieu virtuellement le 29 janvier 2021. Pendant deux heures, Laura Cahier (depuis la France) et Laetitia Braconnier Moreno (depuis la Colombie) ont discuté avec Armstrong A. Wiggins (depuis Washington, D.C.) de son voyage personnel, de son histoire et de son implication dans les luttes pour les droits des Peuples autochtones, depuis le milieu des années 1970 jusqu'à aujourd'hui. De la criminalisation aux attaques systémiques en passant par d'autres formes de discrimination, Armstrong explore, dans cet entretien, les difficultés auxquelles continuent d'être confrontés les leaders autochtones des Amériques, dans le cadre de la mise en œuvre de leurs droits. Ses propos détaillent, entre autres, la façon dont les luttes menées par les Peuples autochtones pour leurs droits humains se situent en relation - voire, en contraste - avec d'autres mouvements sociaux. Plaidant pour une éducation universelle aux droits des Peuples autochtones, il se livre à un exercice de pédagogie sur leur culture juridique, en mettant l'accent sur leurs droits collectifs à l'autodétermination, à la terre, au territoire, aux ressources naturelles, entre autres. Laetitia Braconnier Moreno et Laura Cahier tiennent adresser leurs remerciements les plus chaleureux à Armstrong pour leur avoir accordé cet entretien. Elles ont également apprécié les commentaires critiques et le soutien apportés par Miranda Carman dans le cadre de la retranscription de cet entretien. ${ }^{2}$

Photo 1 : Armstrong Wiggins à Washington, D.C. avec le Capitole étasunien en arrière-plan, 2012

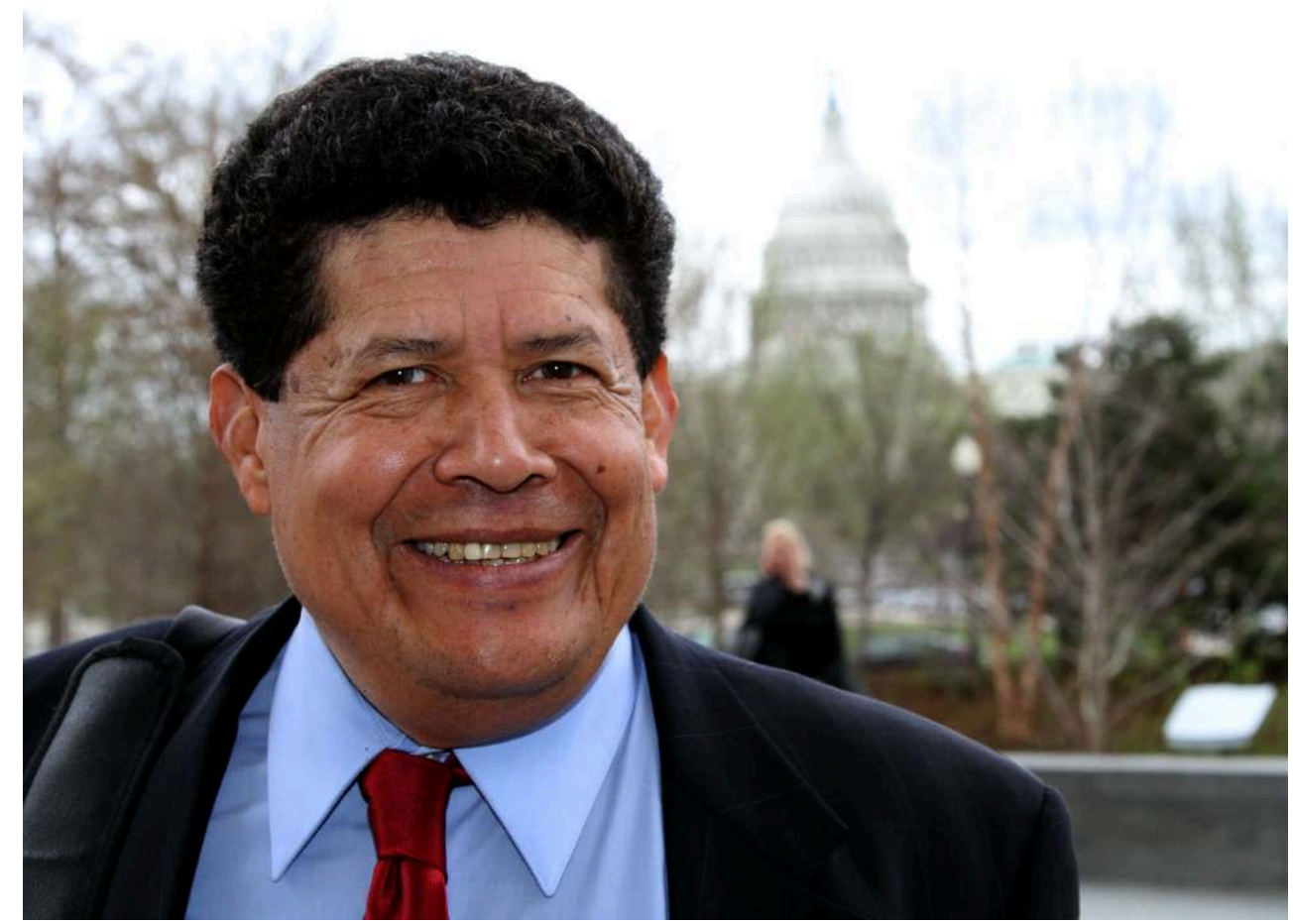

Crédits : Armstrong A. Wiggins 
Laura Cahier \& Laetitia Braconnier-Moreno (L.C. \& L.B.M.) : Merci, Armstrong, d'avoir accepté de nous rencontrer aujourd'hui. Nous sommes ravies de discuter du sujet des défenseurs des droits humains dans les Amériques, en nous basant sur votre propre expérience et votre parcours. Pour commencer cet entretien, pourriez-vous nous parler de vous et du parcours personnel qui vous a conduit à devenir un défenseur des droits humains?

Armstrong Wiggins (A.W.) : Je m'appelle Armstrong Wiggins. Je suis un Indien Mískito du village de Karatá, sur la côte atlantique du Nicaragua, en Amérique centrale. Je suis sûr que les gens qui liront cet entretien se demanderont : " Comment se fait-il qu'un Indien Mískito, né dans le village de Karatá, sur la côte atlantique du Nicaragua, ait un nom comme Armstrong Wiggins ?» Eh bien, il y a une histoire et un récit derrière ce nom. Vous voyez, dans notre village, il n'y a pas de médecin, mais seulement des sages-femmes pour aider les femmes à mettre leurs bébés au monde. Ma mère a eu des difficultés à me donner naissance et la sage-femme du village n'a pas pu l'aider. Heureusement, il y avait une personne étatsunienne qui travaillait dans notre village, qui avait reçu une formation de médecin, et qui a ainsi aidé à lui sauver la vie. C'est pourquoi mes parents ont décidé de me donner son nom de famille, Armstrong. Comme j'étais bébé, je ne pouvais pas dire «non » à un nom de famille étatsunien! Et mon nom de famille, Wiggins, est lié à l'histoire du protectorat britannique sur la côte atlantique du Nicaragua. Vous voyez, les Nations autochtones Mískito n'ont jamais été colonisées par l'Espagne, ni ne faisaient partie de la République indépendante du Nicaragua jusqu'à ce que l'Angleterre et le Nicaragua négocient le Traité de Managua et Harrison-Altamirano, sans le consentement des Nations Mískito.

Je viens d'un petit village de 27 maisons appelé Karatá ; le village de ma mère était comme un paradis, avant les ouragans Eta et Iota, situé entre une lagune et la mer des Carailbes. Mon père venait du village de Wawa, tout près de celui de ma mère, sur la côte des Carailbes également. Karatá et Wawa ont fait l'objet de reportages ces derniers temps parce que deux puissants ouragans de catégorie 4 ont détruit les deux villages. ${ }^{3}$ Mes familles se battent actuellement pour survivre et reconstruire les deux villages. Cela prendra un certain temps, mais nous les reconstruirons à nouveau. En tant qu'autochtones, nous étions très pauvres d'un point de vue matériel, mais très riches spirituellement et avec beaucoup de ressources naturelles. Dans le passé, nous ne pouvions accéder qu'à une école primaire dans mon village. Le gouvernement nicaraguayen ne s'intéressait pas à l'éducation des Indiens Mískito. Je me souviens que nous écrivions sur des ardoises parce que nous n'avions ni livres, ni papier. Nous allions à l'école chez ma professeure, car il n'y avait pas de bâtiment scolaire dans le village. En raison du manque de place dans sa maison, certains d'entre se mettaient sous le lit pour l'écouter, afin d'apprendre.

Mon père est mort quand j'avais huit ans ; ma mère voulait que je reste dans mon village pour l'aider à s'occuper des animaux. En fait, elle ne voulait pas que j'aille à l'école. Sa philosophie était que si j'allais à l'école, je devrais alors me préoccuper de tous les problèmes de notre communauté, et plus seulement des problèmes de notre famille. Mais, si je n'allais pas à l'école, elle pensait que personne ne me dérangerait pour résoudre les problèmes de la communauté ni me demanderait de devenir un leader, parce que nous étions tous les mêmes. Maintenant, en y repensant, ma mère avait raison : j'aurais dû rester au lieu d'aller à l'école, mais ce n'était pas mon destin. Après la primaire, je ne pouvais plus continuer l'école dans mon village. À ce 
moment-là, pour une raison quelconque, ma professeure a vu en moi du potentiel, surtout en sciences, en philosophie et en histoire. Elle a convaincu ma mère car ils m'avaient trouvé une place dans une école loin de mon village - même s'il n'y ait pas de place pour moi pour être logé et nourri dans la ville de Puerto Cabezas. J'avais huit ans à l'époque. J'avais l'habitude de dormir dans un marché et d'aller à l'école pendant la journée. Ma mère me déposait chaque lundi matin dans un port appelé Lamlaya, puis je marchais seul pendant 45 minutes jusqu'à Puerto Cabezas. Elle revenait me chercher tous les vendredis. Ensuite, elle et moi pagayions en canoë le long des rivières et des lagunes pour retourner à Karatá.

Mais, quand j'étais en sixième, je suis tombé très malade. J'ai fini par me retrouver dans un hôpital de missionnaires moraves. La facture de l'hôpital était cependant très chère pour moi, car je n'avais pas d'argent. J'ai dû négocier avec le directeur de l'hôpital pour travailler afin de payer cette facture. C'est ainsi que j'ai rencontré le Docteur Ned Wallace, directeur de l'hôpital, ingénieur électricien de formation, médecin et ancien chirurgien de la marine américaine. Il a alors accepté que je travaille à l'hôpital afin de payer ma facture. Plus tard, je suis devenu l'un des quatre " garçons d'hôpital » avec le gîte et le couvert à l'hôpital. Grâce à cela, j'ai pu faire mes études secondaires. Comme le Dr Wallace était un ingénieur, un chirurgien et un très bon politicien, il me formait à toutes ces compétences pendant que j'allais au lycée. Il m'a accueilli comme son fils et m'a enseigné de nombreuses compétences en plus de celles propres au domaine de l'électrique. A quatorze ans, j'avais déjà un diplôme en ingénierie électronique du Cleveland Institute of Electronics par correspondance. Le Dr Wallace était mon professeur; il a également payé pour l'école par correspondance, l'enseignement à distance. Je suis ensuite devenu le responsable du système électrique, téléphonique, radio et électronique de cet hôpital de 45 lits. En plus de nettoyer les sols de l'hôpital, de peindre, de couper l'herbe, de nourrir les cochons et les poulets, je m'intéressais aussi à l'ingénierie et au pilotage des avions... J'ai toujours rêvé de piloter des avions et d'aller sur la Lune. Quoi qu'il en soit, je suis allé à l'école à l'étranger pour poursuivre ma première carrière dans l'ingénierie électrique. 
Photo 2 : Carte du Nicaragua, indiquant les villages de Wawa et Karratá

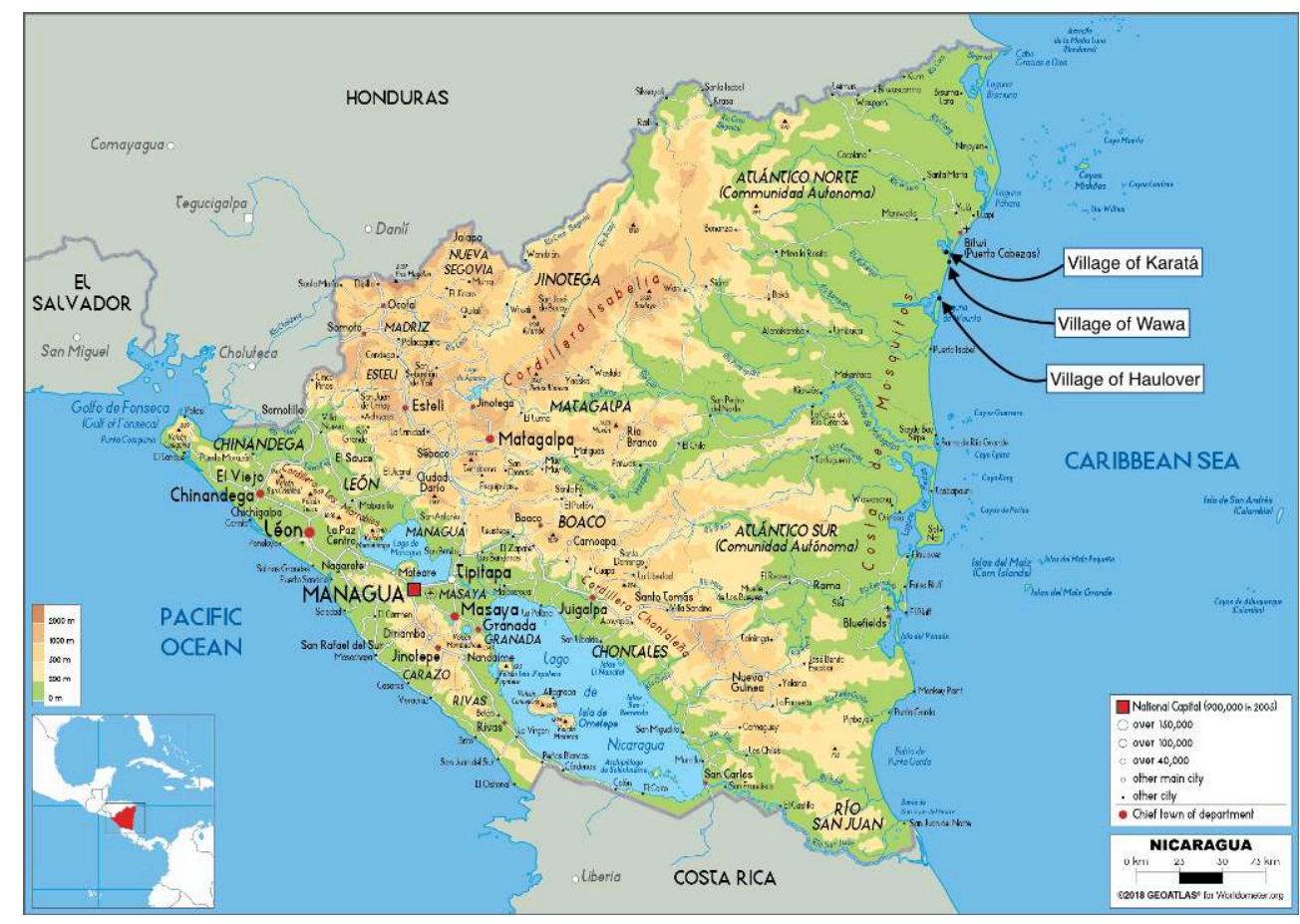

Source : https://www.worldometers.info/maps/nicaragua-map/ (les villages de Wawa, Haulover, et Karatá ont été ajoutés sur la carte par les auteures).

Mais, un jour, j'ai reçu un appel de ma communauté : ils m'ont demandé de revenir au Nicaragua pour travailler avec le programme médical de l'Université du Wisconsin. Ils m'ont proposé d'être directeur de la communication radio et de diriger leur programme de médecine préventive avec des étudiants en médecine et des résidents des Big Ten Universities ${ }^{4}$, afin d'étudier la médecine tropicale sur la côte atlantique du Nicaragua. Bien que j'aie également reçu une offre de l'armée américaine à l'époque, cette demande de ma communauté était importante pour moi. J'ai décidé de revenir au Nicaragua après avoir été loin de chez moi. C'est ce voyage qui a changé ma vie, et je suis ainsi passé de la science à la défense des droits des autochtones et à la politique autochtone. Tout au long de notre travail sur la médecine préventive, nous avons formé des responsables autochtones de la santé, dans les villages de la côte atlantique du Nicaragua. À un moment donné, nous avons décidé d'examiner plus en profondeur la situation de nos propres Nations Mískito : comment pourrions-nous nous éduquer afin que les missionnaires, anthropologues ou autres ne parlent plus en notre nom ? Nous voulions parler pour nous-mêmes, en tant que Peuples autochtones. C'est ainsi que je me suis profondément impliqué dans ces questions non seulement les questions culturelles, mais également nos droits politiques et économiques. Nos droits à l'autodétermination pour gouverner notre peuple. Nos droits à la terre et aux territoires. Notre droit d'envoyer nos jeunes à l'école - pas seulement à l'école primaire et secondaire, mais aussi vers l'enseignement supérieur. Notre droit d'avoir nos propres universités sur la côte atlantique du Nicaragua. C'est devenu mon rêve... Et j'ai abandonné le rêve d'aller sur la Lune.

En 1977, j'ai reçu un appel d'un jeune avocat du nom de Robert T. Coulter ${ }^{5}$, d'un institut amérindien travaillant avec les Six Nations en Amérique du Nord. Ils m'ont demandé de venir avec eux pour parler de nos problèmes fondamentaux en matière 
de droits humains, pour la première fois aux Nations Unies à Genève. J'étais très jeune à l'époque, mais j'étais heureux de participer parce que notre gouvernement le gouvernement du Nicaragua - ne nous représentait pas, ni ne reconnaissait nos droits. Il était très important pour moi de comprendre l'ancien monde appelé Europe, et comment les pays européens, comme l'Angleterre, l'Espagne, le Portugal ou la France, vinrent aux Amériques pendant la période de colonisation, manquèrent de respect à notre civilisation, détruisirent nos systèmes politiques et juridiques existants, et nous transformèrent en leurs esclaves... nous désignèrent comme moins que des gens, plutôt comme des sauvages. Ils ne nous considérèrent pas comme des êtres humains : ils ne reconnurent pas notre civilisation et ils voulurent la détruire par de «gros mensonges» (big lies). C'est pourquoi nous n'acceptons pas qu'on nous appelle "personnes ethniques ». Nous étions de fièrs Nations dans les Amériques avant la colonisation : nos gouvernements existaient et nous avions nos propres lois. Nous croyons aux droits humains individuels et collectifs. Aux Nations-Unies, nous avons travaillé dur pour sensibiliser les Européens et les autres nations, y compris le gouvernement chinois, à nos lois et à nos modes de vie. La Chine a reconnu que nous étions des Nations qui avaient été détruites par les colons européens. Cependant, elle ne voulait pas que ses propres peuples soient appelés « Nations » ou « Peuples », mais plutôt « groupes ethniques ». Le gouvernement de l'Inde a fait valoir le même argument.

Lorsque nous sommes allés pour la première fois à Genève en 1977, nous avons pensé qu'il était temps pour nous de prendre la parole parce que nous n'étions pas reconnus en tant que Peuples. Nous n'avions pas d'autre endroit où aller qu'aux Nations Unies dans le but de lutter pour nos droits humains fondamentaux. Sensibiliser les États membres au fait que nous avons le droit à l'autodétermination en tant que Peuples. C'est pourquoi nous ne sommes pas seulement des mouvements sociaux, car nous luttons toujours pour que nos droits fondamentaux soient reconnus comme ceux des autres êtres humains. Les mouvements sociaux parlent d'équité, de droits culturels, d'éducation - c'est bien, mais nous, en tant qu'Indiens, n'avons pas encore obtenu ces droits là et nous les revendiquons toujours. En fait, je pense que les gouvernements adorent cela, parce qu'ils refusent d'entendre parler de droits humains, de discrimination, d'autodétermination. Les gouvernements préfèrent travailler avec les ONG sur des questions sociales, plutôt que sur les problèmes de droits humains. Pour nous, les droits de l'homme sont des droits légaux, c'est-à-dire des droits légaux à la terre, aux ressources, à l'environnement, ou à gouverner nos propres peuples en tant que Nations.

Avant d'aller à Genève en 1977, je pensais que j'étais seul à me battre pour nos droits sur la côte atlantique du Nicaragua... Je ne connaissais personne d'autre qui se battait pour ces questions. Lorsque je suis allé à Genève pour la première fois, des bus scolaires sont venus de toute l'Europe pour voir les « Indiens ». C'était fou ! Nous ne pouvions même pas sortir de nos hôtels parce qu'ils bloquaient la route. Nous avons commencé à parler de l'élaboration de normes internationales pour les droits des Peuples autochtones. À l'époque, même les ONG étaient opposées à la création d'un groupe de travail sur les droits des peuples autochtones. Elles voulaient que nous travaillions avec le groupe de travail sur les droits des minorités ou l'esclavage, plutôt que d'avoir notre propre groupe de travail en tant que Peuples. En 1977, nous n'étions pas encore reconnus comme des Peuples ; nous n'étions considérés que 
comme des populations. Aujourd'hui encore, la France et l'Angleterre ne reconnaissent pas que nous sommes des Peuples qui croient aux droits individuels et collectifs. Ce fut une négociation très difficile à surmonter, surtout avec les Européens et les autres. Par conséquent, il nous a fallu de 1977 à 2007 pour obtenir une reconnaissance politique, par le biais de la Déclaration des Nations Unies sur les droits des peuples autochtones ${ }^{6}$. Ce fut une bataille très difficile pour nous d'être reconnus en tant que Peuples. L'une des principales questions auxquelles nous, Peuples autochtones du monde entier, avons dû faire face est la reconnaissance de nos droits à l'autodétermination en matière de terres et de territoires. Nous ne pouvons pas survivre sans terre et sans territoires, en tant que Peuples. C'est pourquoi nous avons décidé de nous battre, car les fils et les filles des colonisateurs croyaient que nous n'avions aucun droit à la terre, à la surface et au sous-sol. Nous y croyons fermement, en particulier dans les Amériques : nos ancêtres habitaient Abya Yala ${ }^{7}$ avant l'arrivée des Européens. Aujourd'hui, les gens - surtout les jeunes commencent à réaliser l'importance de ces droits. En 1977, j'ai réalisé que je n'étais pas le seul à me battre pour nos droits. Cependant, à mon retour de Suisse, j'ai été mis en prison par le régime d'Anastasio Somoza. Ils voulaient m'intimider, parce que j'avais parlé contre le gouvernement nicaraguayen aux Nations Unies. Je peux parler de la criminalisation pendant des années, parce qu'elle est utilisée contre nous et pour nous faire taire quant à nos droits. Pour moi, il est plus facile de gagner des droits, mais il est très difficile de mettre en œuvre ce que nous avons gagné. Malgré tout cela, nous espérons toujours mettre en œuvre la Déclaration des Nations Unies sur les droits des peuples autochtones (2007) et la Déclaration américaine sur les droits des peuples autochtones (2016).

Photo 3 : Photo de groupe des délégués autochtones du monde entier à la fin de la réunion de Genève en 1977

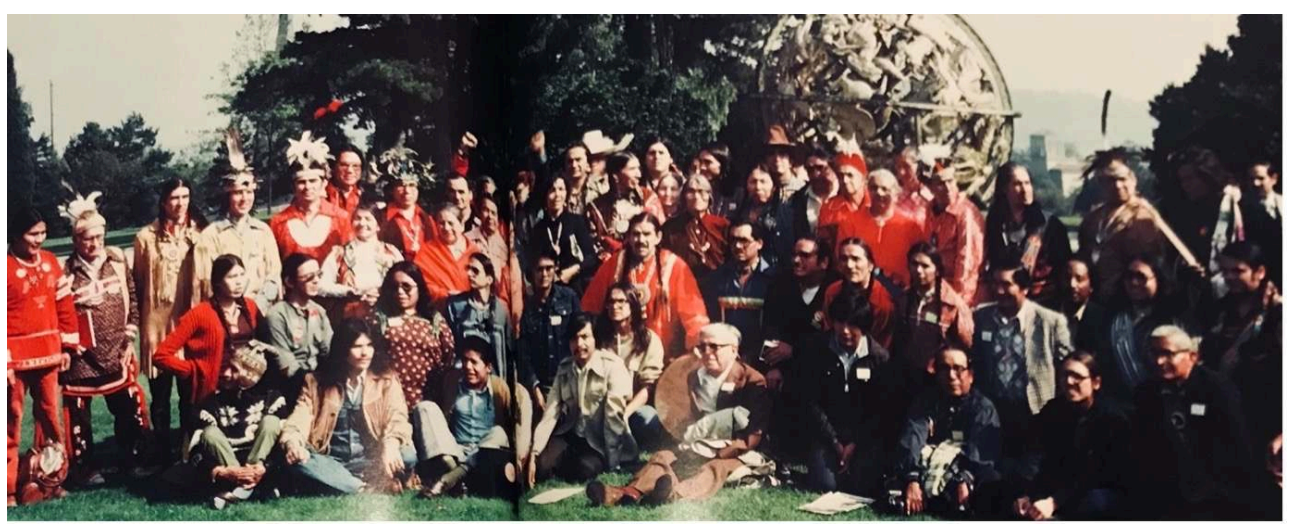

Crédits : Armstrong Wiggins

L.C. \& L.B.M. : Merci de partager votre voyage avec nous. II est instructif de comprendre comment votre histoire personnelle est liée aux luttes pour l'autodétermination et les droits humains. En ce qui concerne plus précisément le Dossier Spécial sur les « líderes sociales » en Colombie, pensez-vous que la terminologie de "leader social » soit appropriée ? En Colombie, par exemple, les leaders autochtones s'intègrent dans la catégorie des «leaders sociaux » pour construire des alliances avec d'autres défenseurs, y compris des leaders paysans ou militantes féministes. Vous considérez-vous comme un « leader social »?

A.W. : Il est vrai que nous devons nous unir pour lutter pour nos droits. Dans un article que j'ai publié dans le Yale Journal of International Law ${ }^{8}$, j'explique comment 
notre lutte se fait non seulement avec les États membres, mais aussi avec certaines organisations de défense des droits humains qui ne s'intéressent qu'aux droits individuels, et non aux droits collectifs. En tant que Peuples autochtones, nous voulons des droits individuels et collectifs. Ceci est lié à l'éducation aux droits humains. La plupart des systèmes d'éducation viennent d'Europe. C'est pourquoi l'organisation pour laquelle je travaille à Washington, D.C., s'appelle l'Indian Law Resource Center (ILRC) ${ }^{9}$. Nous promouvons le droit autochtone, et non le droit européen. Notre objectif est d'éduquer ceux qui n'étudient que les systèmes juridiques coloniaux, mais qui ne connaissent pas notre façon de voir le droit et nos propres systèmes juridiques. Au Mexique, en Amérique centrale, en Amérique du Sud et dans les Caraïbes, le droit autochtone n'est pratiquement jamais enseigné dans les universités, en particulier dans les facultés de droit. Avec l'ILRC, nous avons organisé un programme de formation à l'Université Nationale de Bogota, en Colombie. La plupart de nos étudiants ont levé la main en demandant « pourquoi n'avons-nous pas appris cela avant? » Certains d'entre eux étaient en larmes lorsqu'ils ont appris que la plupart des juges ne connaissaient pas ces questions juridiques.

Quels sont les défis en matière de droits humains ? Les droits de l'homme sont liés à la pauvreté et aux inégalités mondiales. En ce qui concerne les Peuples autochtones, nous sommes au bas de l'échelle - ce que je veux dire, c'est que nous n'avons même pas d'équité. Nous ne possédons pas des maisons à 400000 dollars, nous ne possédons rien en fait - pas même les terres où nous vivions avant la colonisation. En Colombie, ils ne reconnaissent même pas les droits de propriété sur le sous-sol, ils appartiennent tous aux États. Dans de nombreux pays, nous ne pouvons vivre que sur les terres, mais nous ne les possédons pas. C'est pourquoi nous parlons de droits humains, plutôt que de questions sociales. Une deuxième question est liée à la discrimination : les gouvernements préfèrent parler de droits culturels plutôt que de discrimination. Ils préfèrent parler de questions sociales plutôt que d'aborder les véritables problèmes de droits humains liées aux terres et à la discrimination. Ils ne veulent pas parler de conflits armés, de violence, de meurtres de populations autochtones, d'impunité, de déficit démocratique ou de faiblesse des institutions. Pourtant, il s'agit là de droits humains fondamentaux. C'est pourquoi nous sommes des organisations de défense des droits humains, plutôt que des organisations sociales. Cela ne signifie pas que les droits humains et les questions sociales ne se recoupent pas. Nous avons un droit à un environnement sain, à l'éducation et à des systèmes de logement, bien sûr. Cependant, les Peuples autochtones n'en sont pas encore là ! Lors du premier Sommet des Amériques des Peuples autochtones organisé en 2001 à Ottawa, au Canada, le thème était le développement économique. Je me souviens que les dirigeants des Premières Nations du Canada avaient rédigé un projet de déclaration pour le sommet et nous avaient tous demandé de le signer. Cependant, les dirigeants indigènes du Mexique, d'Amérique centrale, d'Amérique du Sud et des Caraïbes ne se sentaient pas à l'aise avec l'idée de signer cette déclaration car elle ne reflétait pas notre réalité - nous luttons toujours pour nos droits les plus basics! Même si nous vivons sur le même continent, le Canada et les États-Unis sont plus avancés dans cette reconnaissance. Les chefs autochtones du Canada ont bien compris que nos réalités étaient différentes. Au Mexique, en Amérique centrale et en Amérique du Sud, nous luttons toujours pour nos droits humains fondamentaux nous n'avons pas encore de droits à la terre... Nous n'avons pas de droits à beaucoup 
de choses, en fait ! C'est pourquoi il y a une différence entre les questions sociales et les questions de droits humains.

Cela ne signifie pas que nous ne pouvons pas travailler avec d'autres organisations sociales. Cependant, les grandes organisations environnementales ne nous comprennent pas $^{10}$. Certaines d'entre elles ne respectent toujours pas les terres autochtones : par exemple, elles développent des zones naturelles protégées, sans consulter les Autochtones qui y vivent. Aujourd'hui, certaines organisations sociales ne veulent pas parler du droit des Peuples autochtones à l'autodétermination, à leurs propres organisations ou à l'autonomie gouvernementale. Contrairement aux organisations sociales, nous avons un droit à l'autodétermination et à l'autonomie. Néanmoins, de nombreuses grandes organisations environnementales ne veulent pas en entendre parler, surtout parce qu'elles reçoivent de l'argent de fonds gouvernementaux, notamment de l'Agence américaine pour le développement international (USAID), de la Banque mondiale, de la Banque interaméricaine de développement (BID) ou des gouvernements européens. Ces bailleurs de fonds gouvernementaux refusent d'entendre parler d'autodétermination et du droit de gouverner notre propre Peuple. Par exemple, au Nicaragua, les organisations environnementales reçoivent des millions de dollars de l'USAID pour travailler sur les questions environnementales, mais elles ne travaillent jamais avec les Peuples autochtones qui vivent réellement sur les terres. Nous continuons à nous battre contre cela. Par exemple, l'ILRC travaille à changer la politique de la Banque mondiale et de la BID à l'égard des Peuples autochtones ${ }^{11}$. Nous faisons des progrès pour améliorer ces normes. Vous voyez, ce sont toutes les raisons pour lesquelles nous sommes différents des organisations sociales - parce qu'elles ne travaillent qu'avec les gouvernements centraux, mais pas avec les Peuples autochtones. Nous aimerions travailler avec les organisations environnementales afin qu'elles puissent contribuer à la création de zones protégées pour les Peuples autochtones, dans lesquelles elles peuvent gouverner. Avec l'ILRC, j'ai en fait créé un manuel sur les droits humains/les droits des Indiens en 1984 pour parler de la législation mexicaine sur les droits fonciers, parce que la plupart des travaux sur les terres autochtones avaient été écrits par des anthropologues qui ne comprenaient pas l'idée de la propriété collective des terres.

L.C. \& L.B.M. : Vous semblez mentionner l'existence de différentes cultures juridiques, notamment entre les systèmes autochtones et occidentaux. Souhaitez-vous partager quelques réflexions sur le thème de la décolonisation du droit ?

A.W. : C'est une question très importante. Avec l'ILRC, nous contestons les lois discriminatoires, en travaillant sur les conflits entre les systèmes juridiques occidentaux et le droit autochtone. Nous travaillons donc sur les réformes du droit dans le but d'éduquer les Écoles de droit, les professeurs de droit et les juges. Bien que la plupart des facultés de droit d'Amérique du Nord - comme Harvard ou Yale enseignent désormais le droit indien fédéral (Federal Indian Law), nous n'en sommes pas encore là en Amérique centrale et du Sud. Nous avons organisé divers séminaires dans la région, notamment au Paraguay et en Colombie avec l'Organización Nacional Indígena de Colombia (Organisation nationale indigène de Colombie, ONIC). Nous avons organisé un troisième séminaire en partenariat avec la Cour régionale des Caraïbes et les écoles de droit de Trinité-et-Tobago et du Guyana. Ce séminaire a été une expérience très riche car des leaders autochtones des Caraïbes y sont venus ainsi que des juges. Nous devons organiser davantage de séminaires sur les lois autochtones, 
mais nous n'avons pas les ressources nécessaires. L'affaire Communauté Awas Tingni contre Nicaragua ${ }^{12}$, que nous avons portée devant la Cour interaméricaine des droits de l'homme, a été la première décision faisant jurisprudence en matière de droits fonciers des autochtones. Lorsque la Convention américaine des droits de l'homme a été rédigée en 1969, les droits fonciers des Peuples autochtones n'étaient pas du tout pris en compte. Dans le texte de cette Convention, il n'est pas question de droits collectifs ; seuls les droits de propriété individuels sont mentionnés. Cependant, nous avons pu utiliser l'article 25 de cette Convention pour justifier de multiples violations des droits humains en lien avec la terre ${ }^{13}$.

Aujourd'hui, les mouvements indiens se renforcent. Nous sommes là ! C'est comme les mouvements de femmes qui disent actuellement : "Nous sommes là ! Vous ne pouvez plus violer nos droits ! » Les Indiens sont devenus des avocats ; ils essaient de contester et de changer les lois. C'est ce que fait l'ILRC : le Centre a été créé par des juristes indiens qui ont d'abord fréquenté les écoles occidentales, mais pour qui cette loi n'avait pas de sens. C'est pourquoi ils ont créé l'ILRC pour représenter nos Peuples, ce sans demander d'argent. Nous ne recevons pas de fonds des gouvernements, nous collectons notre propre argent pour représenter nos Peuples. Nous recevons des directives de nos aînés et de nos leaders - je veux dire, nous ne leur disons pas ce qu'ils doivent faire, mais nous travaillons avec eux pour élaborer des plans juridiques. C'est tout à fait différent de l'attitude de nombreux avocats qui croient connaitre le droit indien et disent à nos leaders comment s'y prendre. Nous devons travailler ensemble et nous éduquer les uns les autres. Nous avons besoin de nos droits légaux d'abord - c'est pourquoi nous travaillons pour nos droits à l'autonomie, à la terre, aux territoires, aux ressources naturelles et à la survie culturelle.

L.C. \& L.B.M. : Au cours de votre travail avec I'Indian Law Resource Center (ILRC) et de votre propre expérience, avez-vous remarqué une forme quelconque de criminalisation des leaders autochtones par les États? Si oui, quelles mesures le ILRC a-t-il prises pour traiter cette question et protéger ces leaders?

A.W. : Avec l'ILRC, nous avons un projet qui traite de la situation des leaders autochtones au Brésil. Dans les années 1980, lorsque nous avons représenté le peuple Yanomami pour la première fois au Brésil ${ }^{14}$, il n'y avait pas d'avocats indiens. Nous avons réussi à collecter des fonds pour aider Paolo Celso Pankararu à devenir l'un des premiers avocats autochtones du pays. Aujourd'hui, la situation est différente au Brésil, car il y a des dizaines d'avocats autochtones. Comme ils sont experts dans leur propre droit national, nous les formons plutôt aux systèmes internationaux des droits de l'homme - le système interaméricain des droits de l'homme, le système des droits de l'homme des Nations unies. Lorsqu'ils n'ont pas de justice dans leur propre pays, nous les formons pour qu'ils puissent accéder à la justice aux niveaux régional et international. En même temps, nous travaillons avec le système interaméricain pour demander des mesures conservatoires afin de protéger les dirigeants autochtones qui sont criminalisés, menacés ou tués. De nombreux assassinats ont lieu au Brésil, en Équateur, au Pérou, en Colombie, au Nicaragua ${ }^{15}$, entre autres pays. Regardez ce qui se passe en Colombie contre les leaders autochtones et afro-caribéens : ils les tuent!

Nous organisons également ce type de formation pour les exposer au niveau international : il est très difficile de tuer des dirigeants autochtones lorsqu'ils sont 
connus à l'étranger, par la communauté internationale. Lorsqu'Obama a été élu Président pour la première fois, j'ai fait venir des dirigeants autochtones d'Amérique centrale et du Sud à la Maison Blanche pour qu'ils rencontrent son service juridique. Nous avons parlé de la criminalisation des leaders autochtones dans tous les pays de la région, et nous avons discuté de la manière dont les États-Unis pourraient contribuer à leur protection. Il ne s'agissait pas seulement de protéger les dirigeants autochtones, mais aussi les organisations des droits humains et les organisations sociales qui luttent pour différentes questions et qui sont harcelées et menacées. Une des avocates noires du département juridique m'a demandé d'expliquer ce que j'entendais par « criminalisation des activistes autochtones ». J’ai répondu : « Eh bien, je vais vous le dire très rapidement. Vous souvenez-vous de l'époque où Martin Luther King se battait pour les droits aux États-Unis et où ils voulaient le tuer ? C'est de cela dont je parle ». Elle m'a dit : « N'allez pas plus loin, je comprends maintenant, je comprends! » Voilà ce qu'il en est. Nous devons travailler tous ensemble. J'espère que, dans les écoles de droit, ils commencent à parler de ça! Nous avons vraiment besoin d'apprendre les uns des autres et de travailler ensemble.

L.C. \& L.B.M. : Vous avez mentionné les mesures conservatoires accordées par la Commission interaméricaine des droits de l'homme pour protéger les leaders autochtones. C'est un exemple des outils juridiques qui existent au niveau international pour protéger les défenseurs des droits humains. Plus généralement, pensez-vous que le système international des droits de l'homme offre des garanties suffisantes pour protéger ces derniers, lorsqu'ils sont confrontés à la criminalisation et à d'autres menaces concernant leur droit à la vie?

A.W. : C'est encore très difficile. J'aimerais pouvoir dire que le système international est très efficace pour protéger les leaders autochtones. Mais il ne l'est pas, notamment en raison de l'attitude de certains États et des fortes divisions politiques existant au sein des institutions internationales, comme l'Organisation des États américains (OÉA). Certaines de ces divisions reflètent les problèmes politiques et idéologiques qui subsistent dans des pays qui ne se soucient pas des droits fondamentaux de leur population. La situation est encore très compliquée dans de nombreux pays, dont le Nicaragua, le Brésil, la Colombie ou le Pérou. Par exemple, même si la Commission interaméricaine des droits de l'homme octroie des mesures conservatoires pour protéger les dirigeants autochtones et les défenseurs des droits humains, certains États membres ne les respectent pas. Cependant, nous ne pouvons pas abandonner! Nous devons continuer à pousser et à dénoncer les gouvernements. À cet égard, la communication est très importante pour éduquer la communauté internationale sur ces questions et pour faire pression sur les gouvernements.

L.C. \& L.B.M. : En ce qui concerne l'importance de la communication publique et de l'exposition médiatique, l'ILRC a travaillé avec les leaders autochtones de la Coordenação das Organizações Indígenas da Amazônia Brasileira (Coordination des organisations indigènes de l'Amazonie brésilienne, COIAB) au Brésil. En 2019, ils ont lancé une campagne internationale pour dénoncer la criminalisation et l'assassinat de leaders dans la région amazonienne, appelée "Sang autochtone : pas une seule goutte de plus ${ }^{16}$. Lorsque les leaders autochtones de la COIAB sont venus à Washington, D.C. en septembre 2019, I'ILRC a organisé des rencontres avec des membres du Congrès américain, de la Commission interaméricaine des droits de l'homme, des journalistes et d'autres organisations 
autochtones. Plus généralement, comment I'ILRC communique-t-il efficacement sur la nécessité de protéger les leaders et les humains des Peuples autochtones?

A.W. : La communication est essentielle ; il est donc crucial de comprendre comment nous faisons cela depuis Washington, D.C. La pandémie de la Covid-19 a créé une situation très difficile pour les militants autochtones et les organisations de défense des droits humains dans le monde entier. Il est compliqué de travailler comme nous le faisions avant la pandémie. D'ailleurs, lorsque la crise de la COVID-19 a commencé en mars 2020, d'autres membres du ILRC et moi-même avons failli être bloqués au Brésil, parce que nous faisions des ateliers avec des leaders autochtones à Manaus ! Dans l'ensemble, la situation est devenue plus difficile pour les communautés avec la COVID-19 ${ }^{17}$. Nous essayons généralement de faire venir des leaders autochtones des Amériques à Washington, DC. Comme vous l'avez mentionné, en septembre 2019, nous avons fait venir des leaders du Brésil afin de rencontrer des membres du Congrès, du Département d'État américain, des ONG et des organisations environnementales pour les informer de la situation actuelle et des violations des droits de l'homme en cours au Brésil ${ }^{18}$. Nous ne faisons pas seulement cela avec le Brésil, mais aussi avec le Guatemala. Nous avons une affaire que nous allons défendre devant la Cour interaméricaine des droits de l'homme au sujet des droits fonciers du Peuple maya au Guatemala. Nous nous rendrons à San José, au Costa Rica ou bien nous y assisterons de façon virtuelle, en fonction de l'évolution de la pandémie. Nous espérons que la décision sera favorable à la délimitation des terres autochtones au Guatemala, car il existe toujours un apartheid de facto avec une minorité qui gouverne la majorité et un terrible racisme dans le pays ${ }^{19}$. Grâce à cette affaire juridique, nous espérons que les droits fonciers des Indiens seront reconnus - comme nous l'avons fait dans l'affaire Communauté Awas Tingni contre Nicaragua.

L.C. \& L.B.M. : C'est assez impressionnant de voir comment le travail de défense des droits humains des Peuples autochtones est mené simultanément à différentes échelles - locale, nationale et internationale. Comment vous situez-vous dans tout cela?

A.W. : Je me considère comme un « defensor de los derechos de los Pueblos Indígenas " (c'est-à-dire, un défenseur des droits des peuples autochtones). Mon principal objectif a été de travailler pour la défense des droits des autochtones et d'éduquer les gouvernements. J'ai voyagé dans toutes les Amériques, de Saint-Mary en Alaska à la « Terre de feu » en Argentine. Dans les années 1980, lorsqu'il y a eu une guerre entre les nations Mískito et le Front sandiniste, nous avons effectué un important travail de diplomatie $^{20}$. Au même moment, 20000 Mískitos se battaient dans les hauts plateaux du Nicaragua. Mais je n'ai jamais cru aux armes. Depuis mon enfance, j'ai toujours cru que les solutions seraient trouvées par des moyens légaux - et non par la guerre. À l'époque, le président colombien Belisario Betancur m'a beaucoup aidé, en offrant le Palacio de Nariño pour négocier avec les Sandinistes. Trino Morales, qui était le président de l'ONIC en Colombie, nous a également aidés. Nous avons beaucoup d'histoires croisées avec la Colombie ! Je suis reconnaissant envers le peuple colombien, c'est pourquoi il est difficile de voir à quel point il souffre aujourd'hui. J'espère qu'ils s'en sortiront !

L.C. \& L.B.M. : Vous nous avez fait part de moments clés de votre parcours au niveau international. Nous nous demandons si vous pourriez également nous parler de votre expérience au Nicaragua, car vous avez dû faire face à des menaces politiques, juridiques et physiques lorsque vous étiez là-bas, surtout à la fin des années 1970 et au début des 
années 1980. Dans une certaine mesure, il y a des similitudes avec le conflit qui se déroule actuellement en Colombie. Comment avez-vous réussi à surmonter ces difficultés?

A.W. : Nous ne pouvons pas nous battre seuls quand les problèmes sont si graves surtout dans un conflit militaire. Nous avons appris cela au Nicaragua : dix-sept membres de ma famille ont été exécutés par l'armée sandiniste, j’ai été forcé de m'exiler et notre peuple a été envoyé dans des camps de concentration. À l'époque, nous avons dû faire face à l'aspect diplomatique de ce conflit, mais ce n'était pas facile. Lorsque je suis allé à Genève en 1977, j'ai été constamment attaqué par des représentants de l'Union soviétique ou de Cuba lorsque je parlais des problèmes en cours au Nicaragua. Ils m'accusaient d'être un " agent de la CIA », alors que je n'étais financé par aucun gouvernement. La politique de l'ILRC le dit très clairement et explicitement : nous n'acceptons pas d'argent public de la part des gouvernements que ce soit des États-Unis, des pays européens, de l'ONU ou de l'OÉA. Nous collectons notre propre argent auprès de donateurs privés. À l'époque, c'était très difficile. Outre la Colombie, l'Espagne et l'Argentine, je dois dire que le gouvernement du président François Mitterrand s'est vraiment impliqué et nous a soutenus au Nicaragua. Cela nous a vraiment aidés à sauver beaucoup de vies !

Je déteste la guerre. Je n'aime pas la guerre. Je tiens à le dire : la guerre est très destructrice, et elle ne résout aucun problème - en fait, elle l'aggrave. À l'époque, MISURASATA $^{21}$ a dû faire face aux problèmes politiques au Nicaragua parce que 40 000 personnes de notre peuple étaient en exil, surtout au Honduras. Ce n'était pas facile pour nous, car nous luttions à la fois contre le gouvernement sandiniste soutenu par Cuba et l'Union soviétique, et contre l'ancienne dictature militaire de droite Somoza, appelée la résistance nicaraguayenne - ou Contras au Honduras - et soutenue par les États-Unis et l'armée hondurienne. Nous avons eu des difficultés à faire face à cette situation. De nombreux groupes de solidarité soutenaient soit le gouvernement sandiniste, soit les Contras. Notre famille était divisée : à cette époque, la plupart des organisations autochtones croyaient dans les organisations de gauche. Elles ne pensaient pas que la gauche violerait aussi leurs droits humains - elles pensaient que seule la droite pouvait le faire ! La propagande sandiniste était très puissante, soutenant qu'elles étaient pour le peuple, qu'elles étaient révolutionnaires. Je dois vous dire ceci : quand il s'agissait des Indiens, c'était différent. Et maintenant, les dirigeants autochtones commencent à croire ce que nous avions essayé de leur dire dans les années 1980 : la gauche ou la droite, c'est la même chose. Nous pensons que l'influence européenne a été si profonde dans les Amériques qu'il n'y a pas d'idéologie américaine, ni de moyens de développer nos propres démocraties. On peut trouver des exceptions dans certaines démocraties autochtones, notamment dans les communautés isolées comme au Brésil. J’ai beaucoup travaillé avec le Peuple Yanomami au Brésil : ils étaient très isolés dans la forêt amazonienne. Toutes les idéologies - ce que j'appelle les «-ismes », comme le capitalisme, le colonialisme, le marxisme, le léninisme - viennent d'Europe. Il n'y a pas de " - isme» qui ait été développé dans le cadre d'un processus démocratique. Il est donc très difficile de se battre avec la Droite ou la Gauche. Aujourd'hui, cela est également vrai pour le développement économique des Peuples autochtones. Soit, vous traitez avec le capitalisme, même si ce modèle ne convient pas aux Peuples autochtones qui ont un développement économique très durable ; soit vous traitez avec le marxisme, même s'il nuit aussi également aux Peuples autochtones. Nous y réfléchissons : si nous obtenons tous nos droits, à quoi ressemblera notre développement économique ? 
Regardez ce qui s'est passé avec Evo Morales en Bolivie : il a dit qu'il représentait un gouvernement indien, mais il a choisi le système économique marxiste-léniniste. Certains dirigeants autochtones ont parlé des effets néfastes de ces modèles économiques ; ils ont expliqué qu'il n'y a pas de différence pour eux entre un système économique de droite et celui développé par Evo Morales. Les Chiquitanos de Bolivie, en particulier, ont été touchés par ces politiques économiques. Je pense qu'à cause de ces questions idéologiques, les négociations ont été très difficiles au Nicaragua. Lors de la troisième négociation que nous avons eue au Mexique grâce au soutien du président Miguel de la Madrid Hurtado, nous avons passé 24 heures «non-stop » à négocier avec les Sandinistes - qui se disent "révolutionnaires " - pour que les Mískitos soient reconnus comme des Peuples. Il ne s'agissait que du mot « Peuple »! Ceci pour montrer à quel point les différences sont profondes avec les idéologies européennes, y compris les idéologies révolutionnaires. Je pense que c'est différent maintenant parce que les Peuples autochtones ont appris, en Colombie ou en Équateur, que la gauche n'était pas vraiment leur « amie » non plus. Ils commencent à comprendre ce que je disais dans les années 1980 : la gauche et la droite essaient toutes deux de minimiser les droits des peuples autochtones, de leur enlever leurs terres et leurs ressources.

Photo 4

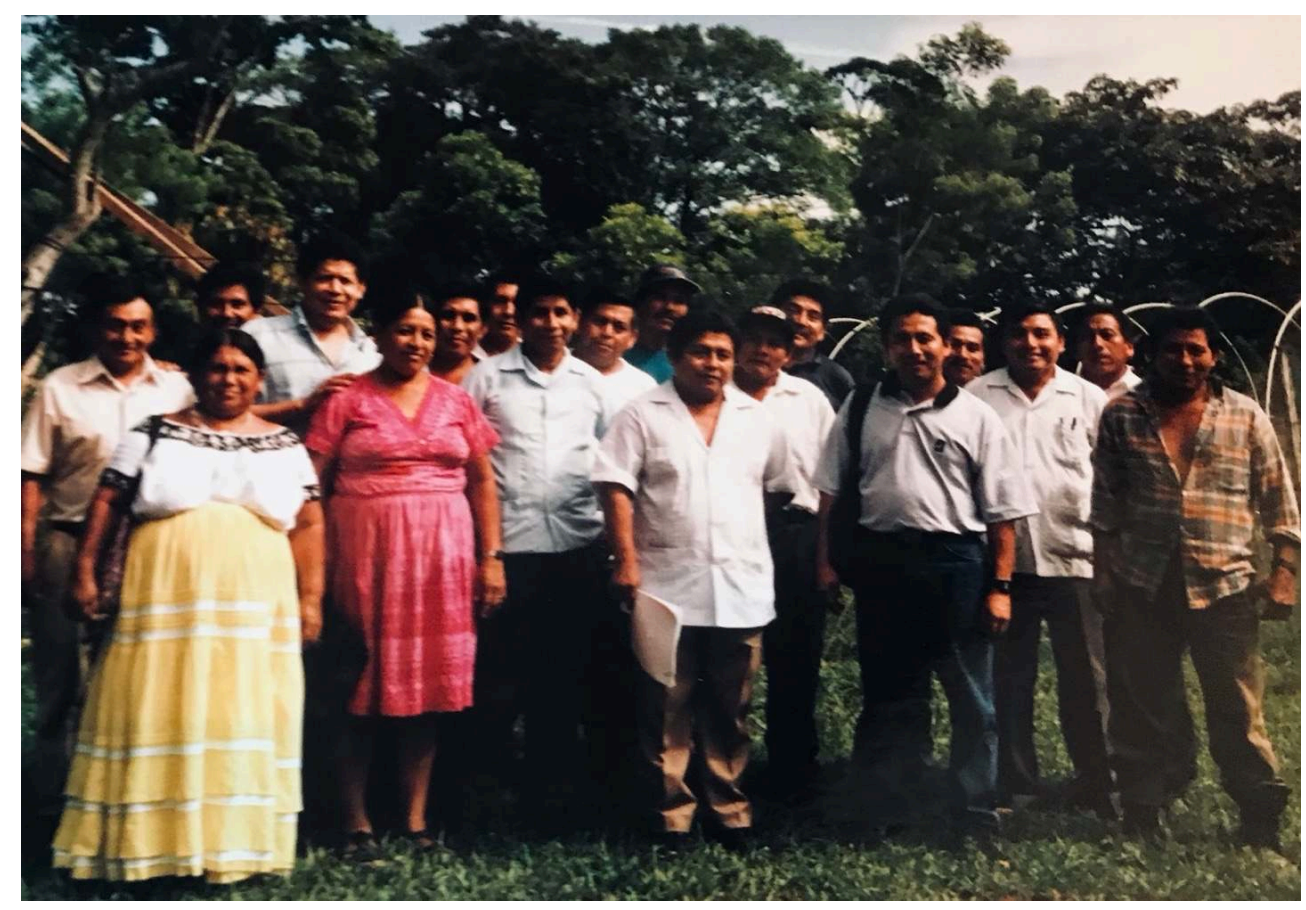

Armstrong Wiggins avec les communautés mayas du Belize au milieu des années 1990 alors qu'ils travaillaient sur les droits fonciers et la création d'un Atlas maya. Voir, Peuple Maya du sud du Belize, Atlas Maya: The Struggle to Preserve Maya Land in Southern Belize, North Atlantic Books, 1997. Avec I'ILRC, Armstrong a contribué à faire avancer le cas des Mayas du sud du Belize, qui réclamaient la reconnaissance de leurs droits à la terre et aux ressources - droits qui étaient complètement niés par le gouvernement bélizien de l'époque. L'affaire a donné lieu à la création d'une loi protégeant leurs droits et a été rapidement adoptée par la Cour suprême du Belize.

Crédits : Armstrong A. Wiggins

Sur la côte atlantique du Nicaragua, nous avons pu obtenir le soutien de l'OEA et négocier les droits fonciers de surface et du sous-sol. Dans les années 1980, la guerre 
était très intense entre les Nations indiennes Mískito et les militaires de gauche sandinistes, et l'OEA est intervenue par le biais d'une mission de maintien de la paix ${ }^{22}$. Nous avons également pu créer des précédents à la Cour interaméricaine des droits de l'homme : dans l'affaire Awas Tingni contre Nicaragua, la Cour a décidé que le Nicaragua devait reconnaître les droits de surface et de sous-sol et créer un mécanisme de démarcation pour délimiter les terres indiennes ${ }^{23}$. Au Nicaragua, nous avons des gouvernements centraux, des gouvernements autonomes régionaux, des gouvernements municipaux et des gouvernements autochtones. Ainsi, nous avons divisé les terres en quatre parties: $25 \%$ des ressources du sous-sol allant au gouvernement central pour les questions d'éducation et de santé, $25 \%$ aux gouvernements autonomes régionaux, $25 \%$ aux gouvernements municipaux et $25 \%$ aux gouvernements autochtones. Cette répartition devait être décidée par les communautés dans le cadre d'une assemblée. C'est là que nous avons également des problèmes. Nous avons ce que nous appelons le droit au consentement libre, préalable et informé, parce que les organisations environnementales ne veulent pas parler du droit à l'autodétermination pour éviter de contester les gouvernements. C'est pourquoi elles ont créé ces choses comme ce droit au consentement en tant que droit légal pour les peuples autochtones. Mais ce sont des droits juridiques secondaires! Les droits primaires sont les droits à l'autodétermination. Nous avons connu des cas où un Indien a donné ou vendu les titres fonciers collectifs à une entreprise, alors que les autres habitants autochtones n'étaient même pas au courant de cette transaction. Il est bon d'être consulté, mais ce n'est pas un droit primaire pour nous - c'est un droit secondaire.

Je suis très préoccupé par la situation actuelle en Colombie. Je ne pense pas qu'il était approprié de négocier à Cuba, étant donné que Cuba était derrière le mouvement de guérilla de gauche. Le problème avec la Colombie est que les Peuples autochtones ont été amenés très tard dans le processus de négociation. Ils ne pensaient pas aux droits des autochtones. C'est pourquoi les peuples autochtones et les descendants des AfroCaraïbes ne sont pas appelés " Nations indiennes », mais « organisations ethniques ». J'espère que les Accords seront mis en œuvre. Je suis la situation de très près, surtout parce que nous travaillons avec le Bureau de Washington sur l'Amérique latine (Washington Office on Latin America, WOLA) à Washington, DC. Nous discutons également avec l'ONIC et d'autres organisations indiennes pour voir comment nous pouvons aider, sur la base de notre propre expérience au Nicaragua, à apporter la paix. Mais c'est très difficile, surtout avec l'administration actuelle en Colombie. J'espère que l'administration de Biden se comportera différemment de celle de Trump avec la Colombie. Il y a tellement de questions idéologiques qui se posent sur les droits des Peuples. J'espère que les assassinats cesseront... Mais je ne sais pas comment cela va finir. Le peuple colombien ne mérite pas de souffrir comme ça.

L.C. \& L.B.M. : Malheureusement, nous voyons se répéter des faits comparables dans différents pays de la région. II est intéressant de noter que les Peuples autochtones ont souvent été associés à la gauche ou à la guérilla au Mexique, en Amérique centrale et en Amérique du Sud. Pensez-vous qu'ils peuvent également souffrir de stigmatisation politique?

A.W. : Vous savez, je suis allé deux fois en prison pour avoir été un activiste autochtone au Nicaragua : une fois sous l'administration de droite du président Anastasio Somoza, et une seconde fois sous l'administration de gauche des Sandinistes. Aujourd'hui, les deux parties ont un certain respect pour moi, car j'ai 
survécu à la prison et j'ai appris de cette expérience. Je pense que c'est pour cette raison que, dans les Amériques, nous devons développer nos propres idéologies - et non pas des idéologies européennes. Nous devons développer nos propres systèmes démocratiques qui fonctionneront pour notre peuple. Cependant, il est difficile de changer cela... Peut-être que la jeune génération commencera à penser comme les peuples d'Abya Yala, au lieu de faire venir cette vieille idéologie du vieux continent. Ces idéologies européennes ne fonctionnent ni ici, ni en Afrique, ni dans le monde arabe. Je ne sais pas si on peut arranger ça... Les gens ont envoyé leurs enfants étudier en Angleterre, en France ou en Espagne, et quand ils reviennent, ils essaient de faire comme en Europe dans les pays d'Amérique - mais cela ne fonctionne pas. En attendant, les puissances coloniales ont emporté toutes les ressources des Amériques vers l'Europe. Les États-Unis ont fait la même chose : ils ont emporté toutes les ressources aux États-Unis, mais ils n'investissent pas dans nos pays et ils ne veulent pas de nous. C'est ce que nous constatons aujourd'hui avec le rejet des migrants en Europe ou aux États-Unis. Les pays riches doivent investir dans ces pays pour les aider à surmonter la corruption, afin que les leaders puissent prendre soin de leur propre peuple. Or, ce n'est pas le cas! Si nous ne changeons pas cela, ce sera un énorme problème, surtout avec l'aggravation de la crise migratoire. Nous devons vraiment avoir un dialogue sur les impacts idéologiques sur les gens - nous devons parler très ouvertement et franchement de la manière de changer cette idéologie.

Ensuite, nous avons aussi la crise environnementale. Regardez ce qui se passe dans les villages de mon père et de ma mère ? Les deux villages ont été complètement détruits par les deux ouragans d'il y a quelques semaines. Les tremblements de terre et les problèmes de pétrole sont préoccupants. Regardez l'oklahoma, il y a des tremblements de terre tous les jours à cause de l'extraction du pétrole. Pourtant, personne n'étudie comment le forage affecte la Terre et les générations futures. Les peuples autochtones ont parlé de ces questions, mais personne ne nous écoute car nous ne sommes pas « assez intelligents " pour comprendre. Mais nous comprenons cela. Je pense donc que l'échange et la discussion sont importants. J'espère que les anthropologues changeront aussi leur façon de penser les questions autochtones. Je me souviens que lorsque j'étais dans mon village, deux anthropologues sont venus d'Allemagne pour faire des études sur les Mískitos. Nous devions leur donner la permission d'étudier dans nos communautés. Nous leur avons dit que nous pouvions le faire, mais nous leur avons aussi demandé si nous pouvions les étudier... Sinon, non : nous ne les accepterions pas dans notre communauté ! La réciprocité est très importante.

L.C. \& L.B.M. : Tout au long de l'entretien, vous avez mentionné le rôle joué par différents acteurs, y compris les États ou les banques de développement, en relation avec les droits des autochtones. Aujourd'hui, qui sont, selon vous, les auteurs de violences contre les dirigeants autochtones et les défenseurs des droits de l'homme?

A.W. : Avant de répondre à cette question, je dois dire quelque chose que j'ai oublié de mentionner plus tôt : les Autochtones sont aussi des êtres humains. Il y a certains cercles vicieux : quand on est pauvre, il peut être difficile de sortir de ces cercles vicieux. Nous avons été utilisés par d'autres forces, comme les sociétés multinationales, pour nuire à notre propre Peuple. Certaines personnes travaillent avec les organisations qui ont fait du mal aux dirigeants et aux communautés autochtones. Ces organisations peuvent être des trafiquants de drogue qui utilisent les terres et territoires autochtones, violant ainsi les droits humains au Mexique, en 
Amérique centrale et en Amérique du Sud. Il peut également s'agir de sociétés minières. Les ressources naturelles sont épuisées à Abya Yala, et celles qui restent se trouvent pour la plupart sur les terres et territoires autochtones. En travaillant avec la communauté Yanomami au Brésil, j'ai vu comment les compagnies minières se comportent. Les compagnies minières avaient l'habitude de tirer sur les Autochtones Yanomami, comme s'ils étaient des singes. Elles les tuaient ou violaient les femmes Yanomami parce qu'elles ne portaient pas de vêtements. Les compagnies minières créent également beaucoup de problèmes dans les territoires indiens : activités de prostitution et trafic sexuel de femmes autochtones, comme au Guatemala ${ }^{24}$. Les sociétés d'exploitation forestière exécutent et tuent également des dirigeants autochtones, comme au Brésil ou en Équateur. Un autre problème en Amérique centrale est l'élevage de bétail. Cette violence se produit également lorsqu'un groupe idéologique se bat pour prendre le pouvoir sur le pays et demande aux Indiens de choisir un camp - et si vous ne le faites pas, ils vous tuent, tout simplement. C'est ainsi que la violence se déroule en Amérique. Je connais plusieurs avocats autochtones de Colombie qui sont aujourd'hui en exil, parce que leur vie a été menacée. La criminalisation se produit partout dans les Amériques! Ils leur font ce qu'ils font aussi avec l'environnement : la destruction.

L.C. \& L.B.M. : Pour conclure sur une note plus positive, vous avez mentionné le travail mené par I'ILRC pour développer des instruments juridiques à I'ONU et à I'OEA dans le but d'améliorer la protection des leaders autochtones. Selon vous, quelles sont les étapes importantes pour avancer et renforcer la protection des leaders et des communautés autochtones dans le futur?

A.W. : Aujourd'hui, la technologie s'est tellement développée que les " mauvaises » organisations ou les États membres ne peuvent plus ignorer les violations des droits humains comme ils le faisaient dans les années 1980 . Nous sommes de plus en plus nombreux à nous instruire et à savoir comment chercher de l'aide. Les jeunes générations deviennent également plus ouvertes à ces questions - qu'elles viennent de France, d'Angleterre, des États-Unis ou du monde entier. Elles reconnaissent mieux les droits des Peuples autochtones, les droits des minorités et les droits des femmes. Nous nous rassemblons, même aux États-Unis : les mouvements de femmes, Black Lives Matter, les mouvements autochtones se rassemblent tous! Nous pouvons maintenant parler au Président, aux Sénateurs, aux Membres du Congrès - et ils répondent à nos demandes. Ce sont des signes d'espoir. Avec l'ILRC, nous continuons à organiser des formations et des ateliers avec les leaders autochtones, et nous partageons également des informations. Et, regardez-vous : vous vous intéressez à ces questions! Je suis sûr que vous continuerez à travailler sur ces questions et à écrire à leur sujet. Nous pouvons maintenant venir en Europe pour parler des enjeux climatiques et nous rendre à New York pour participer au Forum permanent des Nations Unies sur les questions autochtones. La Commission interaméricaine des droits de l'homme et la Cour interaméricaine des droits de l'homme s'occupent également de nos questions. Aujourd'hui, lorsqu'il n'y a pas de justice à l'intérieur du pays, nous la faisons venir de l'extérieur en utilisant les mécanismes internationaux. Même si nous avons encore un long chemin à parcourir, nous allons dans la bonne direction. La Banque mondiale apprend aussi à notre sujet, en faisant des études sur nos terres : elle se rend compte que les Peuples autochtones gèrent mieux les zones protégées que les gens de l'extérieur qui partent une fois qu'il n'y a plus d'argent pour les financer. Cela change, c'est un processus évolutif. J'ai abandonné mon rêve 
d'aller sur la Lune pour un autre rêve : devenir un activiste autochtone et rejoindre la lutte pour la réalisation des droits des Peuples indigènes. Je ne serai pas en vie pour voir ce rêve se réaliser, mais j'espère que la jeune génération poursuivra le travail et le rendra possible.

L.C. \& L.B.M. : Merci, Armstrong, pour vos sages paroles et pour avoir partagé votre voyage avec nous.

Photo 5 : Appel Zoom avec Armstrong Wiggins, Laetitia Braconnier-Moreno, Laura Cahier, 29 janvier 2021

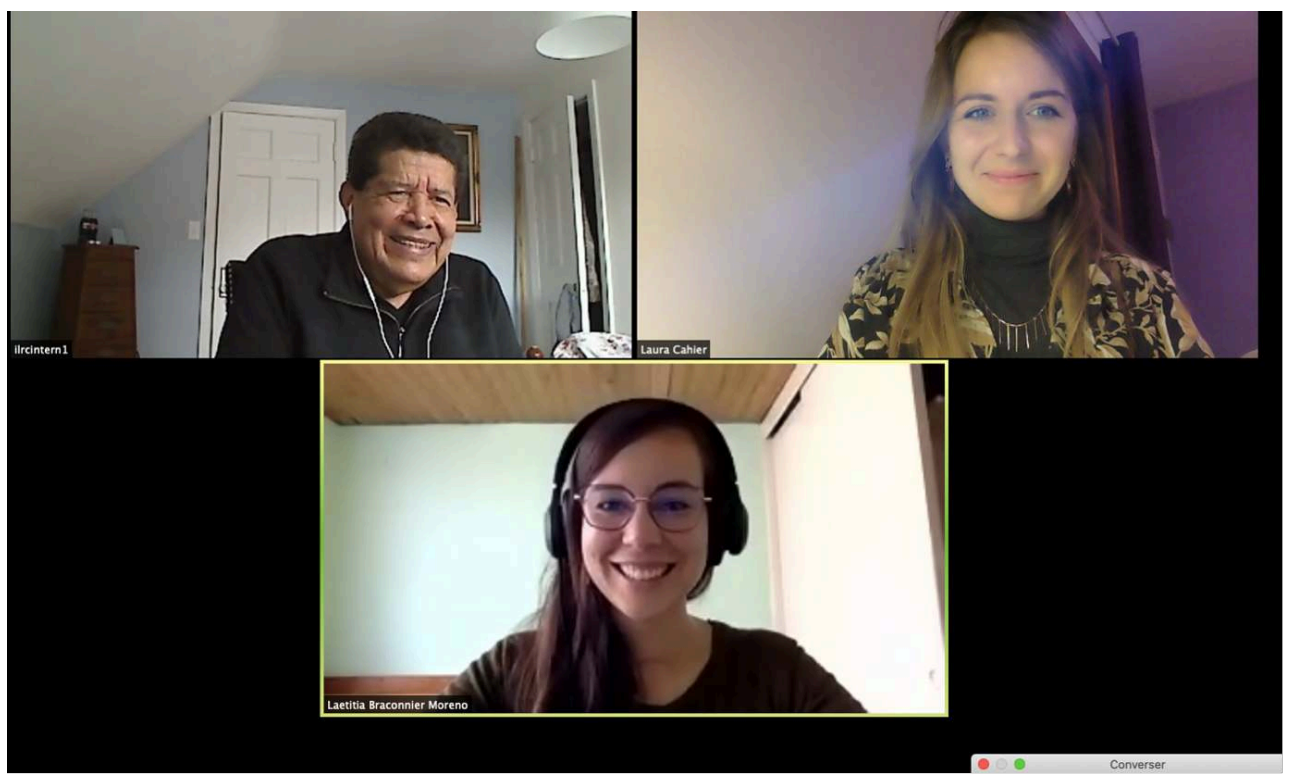

Crédits : Laetitia Braconnier-Moreno \& Laura Cahier

\section{NOTES}

1. Dans la traduction l'entretien de l'anglais vers le français, les auteures ont fait le choix d'adopter une traduction littérale des termes "Indian" et "Indigenous" - soit respectivement "Indien" et "autochtone" - tels qu'employés par Armstrong A. Wiggins.

2. Miranda Carman est l'assistante administrative et de programme de l'Indian Law Resource Center à Washington, DC. Elle est titulaire d'une Licence de l'Université du Nouveau-Mexique et d'une Maîtrise en Études latino-américaines de l'Université de Georgetown.

3. Les villages Mískito de Haulover, Wawa et Karatá ont été détruits par deux ouragans en novembre 2020. À ce sujet, Armstrong note que le changement climatique a des conséquences disproportionnées et dévastatrices pour les communautés autochtones des Amériques, bien que ces problèmes environnementaux soient le plus souvent causés par des activités économiques auxquelles elles ne participent pas. Voir la vidéo du New York Times sur le thème "Rebuild or Leave 'Paradise'" : Climate Change Dilemma Facing a Nicaraguan Coastal Town", disponible à l'adresse suivante : https://www.nytimes.com/video/players/offsite/index.html? videoId=100000007494833. 
4. Les «Big Ten Universities» font référence à dix universités américaines : Ohio State University, Michigan State University, Indiana University, Purdue University, University of Michigan, University of Minnesota, University of Wisconsin, University of Illinois, University of Iowa et University of Nebraska.

5. Robert T. Coulter est autochtone Potawatomi et avocat à Helena, Montana, spécialisé dans le droit amérindien. Il est le directeur exécutif de l'Indian Law Resource Center.

6. Armstrong A. Wiggins a joué un rôle de premier plan dans le travail de normalisation de l'ILRC avec les Nations Unies (ONU) et l'Organisation des États américains (OEA), notamment lors de l'adoption de la Déclaration des Nations Unies sur les droits des peuples autochtones par l'ONU le 13 septembre 2007 et de l'adoption de la Déclaration américaine sur les droits des peuples autochtones par l'OEA le 15 juin 2016.

7. Abya Yala est le nom utilisé par de nombreux Peuples autochtones pour désigner le continent américain. Il est souvent considéré comme un premier pas vers la décolonisation épistémique. Le terme vient de la langue Cuna et signifie « terre en pleine maturité ».

8. Armstrong Wiggins, "Indian Rights and the Environment ", Yale Journal of International Law, Vol. 18, 1993, disponible à l'adresse $:$ https://digitalcommons.law.yale.edu/yjil/vol18/iss1/14/.

9. Pour en savoir plus sur l'histoire de l'Indian Law Resource Center, consulter la chronologie des travaux du Centre depuis 1978, disponible sur : https://indianlaw.org/content/about-center.

10. Voir note de bas de page 8.

11. Depuis 2004, Armstrong supervise les efforts internationaux du Centre en vue de l'établissement de normes dans des organismes internationaux tels que les Nations Unies, l'OEA et les banques multilatérales de développement (BMD), ainsi que la gestion des affaires juridiques pour la protection des droits humains des Peuples autochtones des Amériques.

12. Voir la décision de la Cour interaméricaine des droits de l'homme, disponible à l'adresse suivante : https://www.corteidh.or.cr/docs/casos/articulos/seriec_79_ing.pdf.

13. L'article 25 sur le droit à la protection judiciaire de la Convention américaine relative aux droits de l'homme (1969) stipule que «1. Toute personne a droit à un recours simple et rapide, ou à tout autre recours effectif, devant une cour ou un tribunal compétent pour la protection contre les actes qui violent ses droits fondamentaux reconnus par la Constitution ou les lois de l'État concerné ou par la présente Convention, même si cette violation peut avoir été commise par des personnes agissant dans l'exercice de leurs fonctions officielles. 2. Les États parties s'engagent : a. à faire en sorte que les droits de toute personne qui demande un tel recours soient déterminés par l'autorité compétente prévue par le système juridique de l'État ; b. à développer les possibilités de recours judiciaire ; et c. à faire en sorte que les autorités compétentes fassent respecter ces recours lorsqu'ils sont accordés».

14. En 1979, l'ILRC a porté la première affaire de droits autochtones devant la Commission interaméricaine des droits de l'homme pour la démarcation des terres des Yanomami au Brésil. Tragiquement, le massacre des populations indigènes au Brésil était relativement courant à cette époque. Grâce à l'implication de l'ILRC, les Yanomami ont obtenu une protection et ont pu gérer eux-mêmes leur parc naturel. Voir, http://www.cidh.org/annualrep/84.85eng/brazil7615.htm.

15. Sur la situation au Nicaragua, voir le rapport de la Fédération internationale des droits de l'homme (FIDH), disponible à l'adresse suivante : https://www.fidh.org/es/region/americas/ nicaragua/nicaragua-en-2018-fueron-asesinatos-desde-2019-matan-nuestros? var_mode=calcul.

16. Pour en savoir plus sur la campagne, voir https://en.nenhumagotamais.org.

17. Voir, par exemple, l'article publié par l'ILRC sur les peuples autochtones du Brésil dénonçant les violations des droits humains pendant la pandémie devant la Commission interaméricaine des droits de l'homme, disponible à l'adresse suivante : https://indianlaw.org/brazil/indigenouspeoples-brazil-denounce-human-rights-violations-during-pandemic-inter-american. 
18. Voir, par exemple, l'audience publique organisée à la Commission interaméricaine des droits de l'homme le 27 septembre 2019, https://indianlaw.org/brazil/indigenous-organizationsadvocate-enhanced-protections-inter-american-commission-human-rights.

19. Voir la lettre ouverte écrite par Armstrong A. Wiggins au secrétaire d'État américain John Kerry sur la situation des peuples maya, xinka et garifuna au Guatemala, disponible à l'adresse suivante : https://indianlaw.org/node/988.

20. Après la révolution sandiniste de 1979, Armstrong est devenu le représentant national de MISURASATA, une organisation régionale indienne au Nicaragua.

21. Après la révolution sandiniste de 1979 au Nicaragua, Armstrong devient le représentant national de MISURASATA, une organisation régionale indienne dans le pays.

22. Armstrong Wiggins a aidé les dirigeants indiens Mískito du Nicaragua à obtenir le soutien de la Commission interaméricaine pendant les années de guerre civile entre les Nations indiennes Mískito et le gouvernement sandiniste de gauche des années 1980. L'implication de l'ILRC conduit à des changements dans la Constitution nicaraguayenne en 1987, concernant la reconnaissance des terres et territoires, de l'identité culturelle et des formes d'organisation des peuples indigènes.

23. En 1995, l'ILRC a déposé l'affaire Awas Tingni c. Nicaragua qui a abouti à un arrêt historique de la Cour interaméricaine des droits de l'homme en 2001 pour protéger le droit des Peuples autochtones à la terre, à l'environnement et aux ressources naturelles collectives. La Cour a conclu que l'État nicaraguayen avait violé les droits de la communauté Awas Tingni et a établi que l'État devait adopter dans son droit interne « les mesures législatives, administratives et autres nécessaires pour créer un mécanisme efficace de délimitation, de démarcation et de titrage des biens des communautés autochtones, conformément à leur droit coutumier, leurs valeurs, leurs coutumes et leurs mœurs ". Suite à ce jugement, la Loi 445 - également connue sous le nom de loi du régime de propriété communale des peuples autochtones et des communautés ethniques des régions autonomes de la côte atlantique du Nicaragua et des fleuves Bocay, Coco, Indio et Maiz - a été promulguée en 2003. Cette loi est unique dans la région dans la mesure où elle établit des institutions et des mécanismes de démarcation des terres, de délivrance de titres de propriété et de procédures d'exploitation des ressources naturelles visant à garantir le consentement des communautés autochtones. Voir, Asamblea Nacional de la Republica de Nicaragua, Ley $\mathrm{N}^{\circ} 445$, La Gaceta Diario Oficial, $\mathrm{N}^{\circ} 16$ del 23 de Enero de 2003, disponible sur https://www.poderjudicial.gob.ni/pjupload/costacaribe/pdf/Ley_445.pdf.

24. Voir, par exemple, le rapport thématique de la Commission interaméricaine des droits de l'homme sur les industries extractives et les droits de l'homme (2016), https://www.oas.org/en/ iachr/reports/pdfs/ExtractiveIndustries2016.pdf.

\section{AUTEURS}

\section{LAETITIA BRACONNIER-MORENO}

Laetitia Braconnier Moreno est représentante du pôle Bogota de l'Institut des Amériques. Avocate de l'Université Pontificale Bolivarienne (Medellin) et diplômée d'un Master 2 Droits humains de l'Université Paris Nanterre, elle est Doctorante en cotutelle entre cette Université (UMR 70/74, CREDOF) et l'Université Nationale de Colombie (EILUSOS). Elle est coprésidente de la commission « justice transitionnelle » de l'Association des juristes franco- 
colombiens, et ses travaux portent notamment sur les droits des personnes migrantes et des groupes ethniques, et le pluralisme juridique. lbraconnier.moreno@gmail.com.

\section{LAURA CAHIER}

Doctorante en Droit Public à l'Université d'Aix-Marseille (UMR 7318, CERIC - ED67), chercheuse invitée à l'Université de Georgetown et représentante de l'Institut des Amériques à Washington DC. Diplômée d'un Master en Affaires Internationales (Sciences Po Lyon) et d'un Master 2 en Droits de l'homme et Droit humanitaire (Paris 2 Panthéon-Assas), ses recherches doctorales portent sur les droits des femmes autochtones et leur accès à la justice dans les Amériques. Elle est également membre du groupe de recherche « Missing and Murdered Indigenous Women » à Georgetown University. laura.cahier@gmail.com. 